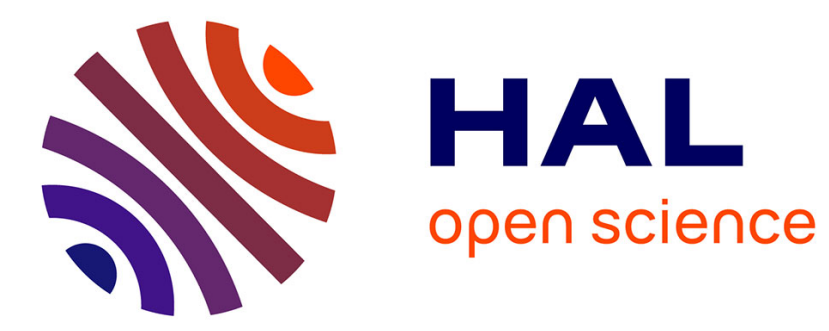

\title{
Reflection of Nonlinear Acoustic Waves from the Mechanically Free Surface of an Unconsolidated Granular Medium
}

Vitali Goussev, Vladislav Aleshin, Vincent Tournat

\section{- To cite this version:}

Vitali Goussev, Vladislav Aleshin, Vincent Tournat. Reflection of Nonlinear Acoustic Waves from the Mechanically Free Surface of an Unconsolidated Granular Medium. 2007. hal-00172558

\author{
HAL Id: hal-00172558 \\ https://hal.science/hal-00172558
}

Preprint submitted on 17 Sep 2007

HAL is a multi-disciplinary open access archive for the deposit and dissemination of scientific research documents, whether they are published or not. The documents may come from teaching and research institutions in France or abroad, or from public or private research centers.
L'archive ouverte pluridisciplinaire HAL, est destinée au dépôt et à la diffusion de documents scientifiques de niveau recherche, publiés ou non, émanant des établissements d'enseignement et de recherche français ou étrangers, des laboratoires publics ou privés. 


\section{Reflection of Nonlinear Acoustic Waves from the Mechanically Free Surface of an Unconsolidated Granular Medium}

(short title: nonlinear reflection from the surface of a granular medium)

$$
\text { V. Gusev }{ }^{1,2} \text {, V. Aleshin }{ }^{1} \text { and V. Tournat }{ }^{2}
$$

1: Laboratoire d'Acoustique de l'Université du Maine UMR-CNRS 6613, Université du Maine, Avenue Olivier Messiaen, 72085 Le Mans cedex 9, France. Vitali.Goussev@univ-lemans.fr

2: Laboratoire de Physique de l'Etat Condensé UMR-CNRS 6087, Université du Maine, Avenue Olivier Messiaen, 72085 Le Mans cedex 9, France

\section{Summary}

The problem of the nonlinear reflection of acoustic waves from a mechanically free surface of an unconsolidated granular layer under gravity is solved analytically using the successive approximations method. The theory revealed specific dependencies of the characteristics of the generated acoustic harmonics of longitudinal and shear waves on frequency and the thickness of the granular layer, which are related to a power-law gravity-induced depth stratification of linear and nonlinear mechanical properties of the granular layer. The developed theory could be useful for the analysis of the acoustic experiments directed to the investigation of fundamental mechanical properties of unconsolidated granular media near the jamming transition taking place at zero confining pressure.

PACS no. 43.25.Jh; 43.25.-x; 45.70.-n; 81.05.Rm 


\section{Introduction}

In the scientific community there exists continuously high interest to mechanical properties of unconsolidated granular materials such as sand [1-3]. In particular, behavior of granular media near the jamming transition at zero pressure, where with increasing pressure the emergence of the solid-like elasticity appears, still contains many puzzles [4-9]. In addition, the low-pressure region near the phase transition to the solid-like state in a granular material is extremely difficult to access experimentally. To our knowledge only the latest optical experiments with the random packing of transparent cylinders [10] have provided first data on the character of growth of the elastic moduli in this medium with increasing pressure in the vicinity of jamming transition. Unfortunately, these experiments are possible in 2D granular medium only.

Acoustical methods are known to be among the most important in characterizing mechanical properties of an unconsolidated granular medium [11-13]. In the currently existing terminology the most important "classical" acoustical experiments in granular medium, consisting in the measurements of the sound velocity dependences on pressure [11,14-18], can be called acousto-elasticity experiments and are considered as nonlinear acoustical experiments $[19,20]$.

Among the "classical" nonlinear acoustical observations in an unconsolidated granular medium is increasing wave absorption (self-induced absorption) and decreasing wave velocity (elastic modulus softening) with increasing wave amplitudes [12]. The observations in granular medium of higher harmonics generation [21] (including processes accompanied by acoustic wave mode conversion [22]), of acoustic wave packet demodulation [23-25], of subharmonics excitation [26], and of modulation transfer [27] have been reported. Nonlinear acoustic methods have been applied for the first time for characterization of the intergrain force statistical distribution [24] which is a fundamental property of granular materials. 
The low-pressure conditions corresponding to the vicinity of the jamming transition are naturally realized near mechanically free horizontal surface of an unconsolidated granular medium. Thus, consideration of nonlinear sound propagating near such a surface could provide a method for testing the behavior of 3D random granular packings near the phase transition to the solid-like state. In this paper we present a theoretical analysis of the nonlinear reflection of acoustic waves from the mechanically free surface of an unconsolidated granular medium. It might be expected that the diagnostic of near surface region of granular materials in acoustic reflection mode could provide information on the state of granular medium near the surface. It is worth mentioning here that another opportunity for testing the near surface region through propagating surface waveguide modes is currently a subject of intensive experimental $[28,29]$ and theoretical $[30,31]$ research.

Our analysis of the wave reflection starts with the fact that gravity induces strong inhomogeneity in the elastic moduli associated with the solid like state of granular materials. Both experiments [14-18] and theory [4-9, 12, 32-34] reveal power-law dependencies of elastic moduli on pressure and, consequently on depth. The peculiar feature of this gravity-induced stratification of a granular material is vanishing of the linear elastic moduli exactly at the free surface of a cohesionless granular material, where in the effective medium approximation the pressure equals zero, and the ratio of the linear modulus to the nonlinear modulus diverges. Consequently, acoustical nonlinearity is expected to play an important role in the reflection process. To simplify the analysis, the discrete nature of granular media leading to scattering and dispersion of acoustic waves will be neglected as well as the linear absorption of acoustic waves. We also do not take into account a possible interaction of the solid-based mode of interest here (transporting energy mainly through the granular skeleton) with an air-based mode (transporting energy mainly through air-filled pores) [25]. The validity of this approximation is expected in the 
vicinity of the mechanically free surface of a granular medium, where the velocity of the solidbased mode is significantly lower than the velocity of the air-based mode, and, consequently, the interaction of two modes is highly asynchronous.

The granular media will be considered as continuous and locally isotropic. For the derivation of the effective macroscopic stress/strain relationship, the information on the microscopic behavior of the individual mechanical contacts between the grains and their orientation is necessary. Henceforth it will be assumed that the grains are arranged in the simple cubic array $[11,35]$ with one of the symmetry axes oriented along the gravity direction. Only the analysis of normal incidence of the plane acoustic waves on horizontal surface of a granular medium will be presented below.

The geometry of the problem is presented in Figure 1. The wave is launched from a homogeneous half-space $(y \geq H)$ to a layer of unconsolidated granular material $(0 \leq y \leq H)$ with the mechanically free surface at $y=0$.

It should be mentioned from the very beginning that the theory developed below is unable to provide a definite reply to the question of possible utility of the method of nonlinear acoustic reflection for diagnostics of granular surface. In this paper we just make a first necessary step towards answering this question and demonstrate how far it is possible to advance analytically in description of nonlinear acoustic phenomena in a macroscopically inhomogeneous medium with the power law variation of linear and nonlinear properties. This analysis does not take into account such important factors as linear absorption of acoustic waves and, most importantly, their scattering caused by the neglected micro-inhomogeneity of a granular medium, which could be extremely important near the surface. Sacrificing these factors, we derive an exact analytical 
solution for the linear reflection problem. Correspondingly, for the nonlinear reflection a mathematical method of successive approximation can be efficiently applied.

Section 2 presents the description of the stress-strain relationships accepted in our theory for the analysis of the acoustic wave propagation. Section 3 describes basic features of linear acoustic waves reflection. Section 4 is devoted to the analysis of the second harmonic generation for the longitudinal acoustic wave and third harmonic generation for the shear wave. In Sections 5 and 6 we present discussions and conclusions.

The preliminary results of our theoretical research have been reported elsewhere [38].

\section{Quadratic approximation of nonlinear acoustics for an unconsolidated granular medium}

To illustrate basic features of the problem we first present the derivation of the stress/strain relationship for the longitudinal acoustic waves in the quadratic approximation of nonlinear acoustics starting from a macroscopic phenomenological model in continuous approximation. Let us assume that the normal stress $\sigma_{y y} \equiv \sigma$ is a nonlinear function $\sigma=\sigma(\varepsilon)$ of normal strain $\varepsilon_{y y} \equiv \mathcal{E}$. In the absence of the acoustic wave, the stress/strain relationship provides $\sigma_{0}=\sigma\left(\varepsilon_{0}\right)$, where $\sigma_{0}$ and $\varepsilon_{0}$ are the static values of stress and strain, respectively. By its definition, an acoustic wave is a weak perturbation $\sigma^{\prime}, \mathcal{E}^{\prime}$ relative to the static values: $\sigma=\sigma_{0}+\sigma^{\prime}$, $\varepsilon=\varepsilon_{0}+\varepsilon^{\prime},\left|\sigma^{\prime}\right|<<\left|\sigma_{0}\right|,\left|\varepsilon^{\prime}\right|<<\left|\varepsilon_{0}\right|$. Thus, in the quadratic approximation of nonlinear acoustics, the stress/strain relationship is presented through its Taylor expansion as

$$
\sigma=\sigma_{0}+\sigma^{\prime} \cong \sigma\left(\varepsilon_{0}\right)+\sigma_{\varepsilon}\left(\varepsilon_{0}\right) \varepsilon^{\prime}+(1 / 2) \sigma_{\varepsilon \varepsilon}\left(\varepsilon_{0}\right)\left(\varepsilon^{\prime}\right)^{2}
$$


Then the equation for the vertical motion of the material $\rho \partial^{2} u_{y} / \partial t^{2}=\partial \sigma_{y y} / \partial y+\rho g$ takes the form

$$
\rho \frac{\partial^{2} u_{y}}{\partial t^{2}}=\sigma_{\varepsilon}\left(\varepsilon_{0}\right) \frac{\partial}{\partial y} \varepsilon_{0}+\frac{\partial}{\partial y}\left[\sigma_{\varepsilon}\left(\varepsilon_{0}\right) \varepsilon^{\prime}+\frac{1}{2} \sigma_{\varepsilon \varepsilon}\left(\varepsilon_{0}\right)\left(\varepsilon^{\prime}\right)^{2}\right]+\rho g
$$

Here $\rho$ is the density, $g$ is the gravity acceleration, and $u_{y}$ is the mechanical displacement along the $y$-axis. From the static part $\sigma_{\varepsilon}\left(\varepsilon_{0}\right) \partial \varepsilon_{0} / \partial y+\rho g=0$ of equation (2) the dependence of the static stress and the static pressure $p$ on depth are found:

$$
\sigma\left(\varepsilon_{0}\right)=-\rho g y=-p(y)
$$

In the expansion (1) the coefficient $\sigma_{\varepsilon}\left(\varepsilon_{0}\right)$ is the local elastic modulus related to the velocity $c_{L}$ of the longitudinal acoustic waves by

$$
\sigma_{\varepsilon}\left(\varepsilon_{0}\right)=\rho c_{L}^{2}
$$

Multiple experiments [14-18, 24] demonstrate that in unconsolidated granular media the velocity of both longitudinal and shear acoustic waves is the power function of pressure (of normal stress) and, consequently, in accordance with equation (3), of depth coordinate

$$
c_{L}=c_{L 0}\left(y / y_{0}\right)^{\alpha}
$$


with typical values of the power $\alpha$ in the interval $1 / 6 \leq \alpha \leq 1 / 3$. The Hertz-Mindlin contact theory $[39,40]$ predicts $\alpha=1 / 6$ both for longitudinal and shear acoustic waves $[32,18]$. The experimental observations of higher powers up to $\alpha=1 / 4$ (both for longitudinal and shear waves) could be theoretically attributed to different factors $[33,34,18]$, such as increasing with pressure number of contacts between a bead and its neighbors, i.e. to an increasing coordination number [18]. Even higher values of $\alpha(\alpha \approx 0.3)$ have been documented but only for shear waves in a very limited interval of experimentally accessible pressures $[15,16]$. However, the power $\alpha=1 / 3$ consistently appears in computer experiments with shear loading near jamming transition [4-9] and can be supported by some analytical arguments [7]. The theories relate faster decrease of the elastic shear modulus with pressure in comparison with the bulk modulus to an additional relaxation of the applied shear stress through a non-affine motion of the beads, which does not influence importantly bulk modulus [4-7].

It should be mentioned that neither the characteristic scale of the wave phase velocity $c_{0}$ nor the characteristic depth $y_{0}$ exist in the ideal limiting case of the non-truncated power-law velocity distribution starting from zero value at the surface and infinitely increasing with depth. The velocity distribution being self-similar depends not on $c_{0}$ and $y_{0}$ separately but on their combination $c_{0} / y_{0}{ }^{\alpha}=c / y^{\alpha}$, which can be calculated theoretically or estimated using experimental data. The normalization introduced in the profile (5) however suits well for the analysis of the cases where the characteristic scale of the velocity is introduced in the model by surface loading or/and internal residual compression of granular material due to adhesion forces between the grains including forces associated with possible interstitial liquid bridges between the grains, or a modification of the power function of rigidity below some critical depth (as it is in the geometry considered in Figure 1, for example). 
The experimental results described by the approximation (5) are consistent with the theoretical formulas (3), (4) if the stress/strain relationship is modeled phenomenologically by the power law

$$
\sigma\left(\varepsilon_{0}\right)=-\bar{\sigma}\left|\varepsilon_{0}\right|^{\frac{1}{1-2 \alpha}}=-\rho g y
$$

where $\bar{\sigma}$ is a positive constant, while stress and strain in granular media are negative. We can conclude that in the vicinity of a mechanically free surface of a granular material

a) the linear elastic modulus decreases with depth, when $\alpha>0$ :

$$
\sigma_{\varepsilon}\left(\varepsilon_{0}\right)=\bar{\sigma} \frac{1}{1-2 \alpha}\left|\varepsilon_{0}\right|^{\frac{2 \alpha}{1-2 \alpha}}=\rho c_{L}^{2} \propto y^{2 \alpha}
$$

b) the nonlinear elastic modulus decreases in magnitude for $\alpha>1 / 4$ but increases in the important for longitudinal waves region $\alpha<1 / 4$ :

$$
\sigma_{\varepsilon \varepsilon}\left(\varepsilon_{0}\right)=-\bar{\sigma} \frac{2 \alpha}{(1-2 \alpha)^{2}}\left|\varepsilon_{0}\right|^{\frac{4 \alpha-1}{1-2 \alpha}} \propto y^{4 \alpha-1}
$$

c) their relative value increases in magnitude for $\alpha<1 / 2$, i.e. in the complete domain $1 / 6 \leq \alpha \leq 1 / 3$ documented for unconsolidated granular media: 


$$
\frac{\sigma_{\varepsilon \varepsilon}\left(\varepsilon_{0}\right)}{\sigma_{\varepsilon}\left(\varepsilon_{0}\right)}=-\frac{2 \alpha}{1-2 \alpha}\left|\varepsilon_{0}\right|^{-1}=-2 \alpha \frac{c_{L}^{2}}{g y} \propto y^{2 \alpha-1} .
$$

Note that the relation $\left|\varepsilon_{0}\right|=(1-2 \alpha)^{-1} c_{L}^{-2} g y$, used in the equation (9), is derived from the comparison of equations (6) and (7). It follows from the relationship (9) that for the range $1 / 6 \leq \alpha \leq 1 / 3$ typical for granular media the ratio of nonlinear to linear modulus diverges when $y \rightarrow 0$, confirming the expected importance of the nonlinear phenomena near the free surface.

This physical nonlinearity is much stronger than the cinematic (geometrical) nonlinearity of the relationship $\varepsilon=\partial u_{y} / \partial y+\left(\partial u_{y} / \partial y\right)^{2} / 2$ between the strain and displacement gradient. So the geometrical nonlinearity will be neglected in the following analysis. As a result the dynamic part of equation (2) describing the propagation of the acoustic waves becomes

$$
\rho \frac{\partial^{2} u_{y}^{\prime}}{\partial t^{2}}=\frac{\partial}{\partial y}\left[\sigma_{\varepsilon}\left(\varepsilon_{0}(y)\right) \frac{\partial u_{y}^{\prime}}{\partial y}+\frac{1}{2} \sigma_{\varepsilon \varepsilon}\left(\varepsilon_{0}(y)\right)\left(\frac{\partial u_{y}^{\prime}}{\partial y}\right)^{2}\right]
$$

with the depth-dependent coefficients in front both of linear and nonlinear terms. In the equation (10) $u_{y}^{\prime}$ denotes the particle displacement in the acoustic wave. The equation (10) can be rewritten in terms of experimental power-law dependence of sound velocity on depth as

$$
\frac{\partial^{2} u_{y}^{\prime}}{\partial t^{2}}=\frac{\partial}{\partial y}\left[c_{L}^{2}(y) \frac{\partial u_{y}^{\prime}}{\partial y}-\alpha \frac{c_{L}^{4}(y)}{g y}\left(\frac{\partial u_{y}^{\prime}}{\partial y}\right)^{2}\right] .
$$


It is instructive to relate the parameters of the nonlinear acoustic model (10), (11) to the parameters of the individual grains in a particular case of spherical grains packed in a simple cubic lattice (array). For the description of the longitudinal plane wave propagating vertically in a simple cubic lattice, the Hertz theory for a contact under the action of normal force is relevant [39]. The theory gives the relative approach $\delta_{y}$ between the spheres as a function of the grain radius $R$, the reduced modulus $K=(4 / 3) E /\left(1-v^{2}\right)$ with $E$, the Young modulus, and $v$, the Poisson's ratio, and the applied normal force $F_{y} \equiv F$ :

$$
\delta_{y}=2 F^{2 / 3} /\left(K^{2 / 3} R^{1 / 3}\right) .
$$

Assuming $F=-4 R^{2} \sigma_{y y}$ and $\delta_{y}=-2 R \varepsilon_{y y}$, we arrive to $-\varepsilon=(-4 \sigma / K)^{2 / 3}$ and to the stress/strain relationship in the form

$$
\sigma=-(K / 4)|\varepsilon|^{3 / 2} H(-\varepsilon)
$$

where $H$ is the step function. Consequently, from the comparison of formula (13) with equations (6) and (7) it follows that in the Hertz model $\left|\varepsilon_{0}\right|=(4 \rho g y / K)^{2 / 3}$, $\rho c_{L}^{2}(y)=\left|\sigma_{\varepsilon}\left(\varepsilon_{0}\right)\right|=(3 / 8) K|\varepsilon|^{1 / 2}=(3 / 2)(K / 4)^{2 / 3}|\sigma|^{1 / 3}$ and

$$
c_{L}(y)=(3 / 2)^{1 / 2}[K /(4 \rho)]^{1 / 3}(g y)^{1 / 6} .
$$

The latter means that $\alpha=1 / 6$ for the longitudinal wave in the Hertz model. 
To propose a model equation for the nonlinear propagation of the plane shear wave in the vertical direction we use Hertz-Mindlin theory $[39,40]$ for the contacts between the spheres under the action of constant normal force $F$ and varying tangential force $T$. The theory predicts hysteresis in the relative tangential displacement $\delta_{x}$ of the spheres

$$
\bar{\delta}_{x}=\left\{1+\left(1-\bar{T}_{\max }\right)^{2 / 3}-2\left[1-\frac{\bar{T}_{\max }+\bar{T} \operatorname{sign}(\partial \bar{T} / \partial t)}{2}\right]^{2 / 3}\right\} \operatorname{sign}(\partial \bar{T} / \partial t)
$$

In the above equation (15) $\bar{\delta}_{x}=\delta_{x} / \delta_{0}$ and $\bar{T}=T / f F$ are the normalized displacement and tangential force, $f$ is the coefficient of static friction, the characteristic displacement is

$$
\delta_{0}=\frac{f(2-v)}{2(1-v)} \frac{F^{2 / 3}}{K^{2 / 3} R^{1 / 3}}=\frac{f(2-v)}{2(1-v)}\left(\frac{\delta_{y}}{2}\right),
$$

and $T_{\max }$ is the amplitude of periodic tangential loading with a single minimum and a single maximum over a period (both of the same amplitude). Equation (15) is valid only for sufficiently weak tangential forces $\bar{T}_{\max } \leq 1$, when there is only partial slip in the contact and no total sliding happens, i.e. the central part of the contact rests always adhered $[39,40]$. The displacement $\bar{\delta}_{x}$ in the equation (15) depends not only on the magnitude of the applied shear force. It is different when the force increases $(\partial T / \partial t>0, \operatorname{sign}(\partial T / \partial t)=1)$ and when it decreases $(\partial T / \partial t<0, \operatorname{sign}(\partial T / \partial t)=-1)$ 
In the limit of weak acoustic loading $\left(\bar{T}_{\max }<<1\right)$ the displacement/force relation (15) can be approximated by retaining only linear and the leading (quadratic in force magnitude) nonlinear terms

$$
\bar{\delta}_{x}=\frac{2}{3} \bar{T}+\frac{1}{9}\left[\bar{T}_{\max } \bar{T}-\frac{1}{2}\left(\bar{T}_{\max }^{2}-\bar{T}^{2}\right) \operatorname{sign}(\partial \bar{T} / \partial t)\right]
$$

The nonlinear terms in the above expansion (17) provide a small correction to the linear relation $\bar{\delta}_{x} \cong 2 \bar{T} / 3$. The latter can be used to approximate the nonlinear terms as a function of force without losing the chosen (quadratic) order of accuracy for the $\delta_{x} / T$ relationship. This leads to the inversion of the displacement/force relationship

$$
\bar{T}=\frac{3}{2} \bar{\delta}_{x}-\frac{3}{8}\left[\bar{\delta}_{x \max } \bar{\delta}_{x}-\frac{1}{2}\left(\bar{\delta}_{x \max }^{2}-\bar{\delta}_{x}^{2}\right) \operatorname{sign}\left(\partial \bar{\delta}_{x} / \partial t\right)\right]
$$

Here $\bar{\delta}_{x \max }$ denotes the maximum of the normalized displacement.

$$
\text { Introducing shear stress } \sigma_{x y} \equiv \tau=T /\left(4 R^{2}\right) \text { and shear strain } \varepsilon_{x y}=\left(\partial u_{x}^{\prime} / \partial y\right) / 2=\delta_{x} /(2 R)
$$

in the acoustic wave, we derive the stress/strain relationship

$$
\tau=\frac{3}{2} \frac{f F}{R \delta_{0}} \frac{\partial u_{x}^{\prime}}{\partial y}-\frac{3}{8} \frac{f F}{\delta_{0}^{2}}\left[\left(\frac{\partial u_{x}^{\prime}}{\partial y}\right)_{\max } \frac{\partial u_{x}^{\prime}}{\partial y}-\frac{1}{2}\left(\left(\frac{\partial u_{x}^{\prime}}{\partial y}\right)_{\max }^{2}-\left(\frac{\partial u_{x}^{\prime}}{\partial y}\right)^{2}\right) \operatorname{sign}\left(\partial^{2} u_{x}^{\prime} / \partial y \partial t\right)\right]
$$


Coefficient in front of the linear term in the form (18) can be identified as the linear shear modulus $\rho c_{T}^{2}$ :

$$
\rho c_{T}^{2}(y)=\frac{3}{8} \frac{f F}{R \delta_{0}}=\frac{2(1-v)}{2-v} \rho c_{L}^{2}(y),
$$

Here equation (16) has been used to reveal the relation between the longitudinal and shear velocities. Consequently, in the Hertz-Mindlin model for the shear wave velocity $\alpha=1 / 6$ exactly as for the longitudinal one. Note that the velocity of linear shear waves does not depend on friction coefficient. The nonlinear modulus in the stress/strain relationship (18) can be defined as the coefficient in front of the square bracket:

$$
-\frac{3}{8} \frac{f N}{\delta_{0}^{2}}=-\frac{4 \alpha}{f} \frac{\rho c_{T}^{4}}{g y}
$$

In accordance with the relation (20) the nonlinear modulus is inversely proportional to the friction coefficient. The relative role of the nonlinear effects increases with decreasing coefficient of static friction. However, it should not be forgotten that the domain of validity of quadratic approximation $\bar{T}_{\max } \leq 1$ shrinks with decreasing $f$. With the stress/strain relationship (18) in hands, the equation describing nonlinear shear waves propagating vertically in a cubic array of spheres takes the form 


$$
\frac{\partial^{2} u_{x}^{\prime}}{\partial t^{2}}=\frac{\partial}{\partial y}\left\{c_{T}^{2}(y) \frac{\partial u_{x}^{\prime}}{\partial y}-\frac{4 \alpha}{f} \frac{c_{T}^{4}(y)}{g y}\left[\left(\frac{\partial u_{x}^{\prime}}{\partial y}\right)_{\max } \frac{\partial u_{x}^{\prime}}{\partial y}-\frac{1}{2}\left(\left(\frac{\partial u_{x}^{\prime}}{\partial y}\right)_{\max }^{2}-\left(\frac{\partial u_{x}^{\prime}}{\partial y}\right)^{2}\right) \operatorname{sign}\left(\frac{\partial^{2} u_{x}^{\prime}}{\partial y \partial t}\right)\right]\right\}
$$

Equation (21) for the propagation of shear waves, derived here in the quadratic approximation of nonlinear acoustic theory, is valid for cubic array of Hertz-Mindlin contacts $(\alpha=1 / 6)$. However, the comparison of equation (21) with equation (11), which has been derived earlier for longitudinal wave for arbitrary power-law distribution of the velocity $c_{L}(y)$, indicates that equation (21) could be also a reasonable model not in the case $\alpha=1 / 6$ only but for arbitrary power-law distribution of shear velocity. This hypothesis is in accordance with the recent successful modeling of static behavior of unconsolidated granular materials $[41,42]$, where the important assumption was the dependence of all elastic moduli on pressure. In quadratic approximation of nonlinear acoustics the static stress/strain relationships proposed in $[41,42]$ reproduce the nonlinear term in equation (11) and predict also an additional nonlinear term proportional to square of the shear strain, if the shear strain exists simultaneously with longitudinal one. The additional term describes the generation of longitudinal waves due to effect of dilatancy $[24,41,42]$. The static stress/strain relationship proposed in $[41,42]$ does not reproduce hysteretic quadratic nonlinear term in equation (21), because hysteresis is a dynamic phenomenon. But the stress/strain law from [41,42] adds in equation (21) a quadratic term proportional to the product of longitudinal and shear strains, which describes the modulation of the shear modulus by longitudinal wave, but does not contribute to self-action of shear waves in the quadratic approximation.

In this study, we will limit ourselves to the initial signals in the form of a pure longitudinal or pure shear wave. In this case the role of the two possible additional nonlinear 
terms in equations (11) and (21) is negligible. Initial pure longitudinal wave can not excite shear wave, since this is forbidden by symmetry of the considered geometry. Initially pure shear wave can excite longitudinal wave through the dilatancy, but the inverse influence of this excited longitudinal wave on shear wave will be cubic in shear wave amplitude and should be neglected in the quadratic approximation of nonlinear acoustics. It is also worth mentioning that even in the case when the initial wave contains both longitudinal and shear components of comparable amplitudes, the role of the considered additional terms in the equations (11) and (21) responsible for the interaction between the waves of different polarization will be strongly suppressed in comparison with the nonlinear terms included in the equations (11) and (21) because of the asynchronism of the interaction. As it is well known (and also can be deduced from the theoretical formula (19)) the local velocities of longitudinal and shear waves are quite different in magnitude.

\section{Reflection of linear acoustic waves}

For the harmonic wave $u(y, t)=\tilde{u} \exp (i \omega t)$ of cyclic frequency $\omega$ the linearized equations (11) and (21) both take the same form of the Helmholtz equation

$$
\frac{\partial}{\partial y}\left[c^{2}(y) \frac{\partial \tilde{u}}{\partial y}\right]+\omega^{2} \tilde{u}=0
$$

Here the notation $\tilde{u}$ is used for the particle displacement either for shear or for the longitudinal wave. The notation $c(y)$ is used for the corresponding wave velocity. For the acoustic field with non-zero amplitude at the mechanically free surface $y=0$, equation (22) describes linear increase of acoustic stress with depth in the vicinity of the surface 
$\rho c^{2}(y) \partial \tilde{u} / \partial y=-\rho \omega^{2} \int_{0}^{y} \tilde{u}\left(y^{\prime}\right) d y^{\prime} \approx-\rho \omega^{2} \tilde{u}(0) y$ (for $y \rightarrow 0$ ). Consequently, accounting for the stress depth profile (6), the acoustic approximation condition $\left|\sigma^{\prime}\right|<<\left|\sigma_{0}\right|$, applied above for the derivation of the wave equation, is valid when $\omega^{2}|\tilde{u}(0)|<<g$. This inequality has a clear physical sense: the acceleration of the grains at the surface should be much lower than the gravity acceleration. The surface particles with acceleration exceeding $g$ could be detached from the material's surface. In this case, The material can not be treated not just as weakly nonlinear, but even as continuous.

It should be also mentioned that the time of the acoustic ray propagation from the depth $y=H$ to the surface $y=0 \quad\left(t_{H}=\int_{0}^{H} d y / c(y)\right)$ is finite: $t_{H}=(1 /(1-\alpha))\left(H / c_{H}\right)$ for the values $1 / 6 \leq \alpha \leq 1 / 3$ typical for unconsolidated granular materials. Only for $\alpha \geq 1$ the power-law velocity profiles are associated with "black holes" $[43,44]$, in which the propagation time towards the surface is infinite. In the latter case, the high frequency acoustic waves (rays), which can not be reflected by the slowly varying rigidity profiles in the volume and could be reflected only by divergent gradients of elastic moduli at the surface, will not be reflected at all, just because they are unable to reach the surface.

It is important that for the power velocity function $c=c_{H}(y / H)^{\alpha}$ with $c_{H}$, the acoustic wave velocity at depth $H$ from the surface, the linearized equation (22) has analytical solutions expressed through special functions $[45,46]$. Introducing normalized co-ordinate $\bar{y}=y / H$ and normalized cyclic frequency $\bar{\omega}=\omega H / c_{H}$, the Helmholtz equation (22) describing propagation of linear acoustic waves along the $y$-axis, can be presented in the form 


$$
\frac{\partial}{\partial \bar{y}}\left[\bar{y}^{2 \alpha} \frac{\partial \tilde{u}}{\partial \bar{y}}\right]+\bar{\omega}^{2} \tilde{u}=0
$$

The exact analytical solution of this equation is:

$$
\tilde{u}=\eta^{-\beta}\left[c_{1} J_{\beta}(\eta)+c_{2} Y_{\beta}(\eta)\right]
$$

Here $J_{v}$ is the Bessel function of the first kind and $Y_{v}$ is the Bessel functions of the second kind (also called Weber's function) [47] of order $\beta=(\alpha-1 / 2) /(1-\alpha), \eta=\overline{\omega y}^{1-\alpha} /(1-\alpha)$ and $c_{1,2}$ are arbitrary constants. It is only the first term in the solution (24) that satisfies the stress-free boundary condition at $\mathrm{y}=0$ (see Figure 1). The corresponding displacement field can be expressed as

$$
\tilde{u}=2^{\beta} \Gamma(\beta+1) \tilde{u}(0) \eta^{-\beta} J_{\beta}(\eta)
$$

where $\Gamma$ denotes the Gamma function [47].

We assume that the displacement field is described by the solution (25) inside the layer $0 \leq y \leq H-0$, while at $y=H+0$ it is composed of the incident wave $\tilde{u}_{i}(H) \exp \left[i k_{i}(y-H)\right]$ and the reflected one $\tilde{u}_{r}(H) \exp \left[-i k_{r}(y-H)\right]$, where $k_{i}=k_{r}=k_{H}=\omega / c_{H}$ is the local acoustic wave number at depth $y=H$. Using the conditions of displacements and stresses continuity at the boundary $y=H$ it is straightforward to find the reflection coefficient of the incident acoustic wave from the near surface layer of granular material. Here for compactness we present the 
results only in the case of a substrate acoustically matched to the granular moduli at depth $H$ (see Figure 1):

$$
R(\omega, H, \alpha) \equiv \frac{\tilde{u}_{r}(H)}{\tilde{u}_{i}(H)}=\exp (-i \varphi), \quad \tan (\varphi)=\frac{2 J_{\beta+1}(\bar{\eta}) / J_{\beta}(\bar{\eta})}{1-\left[J_{\beta+1}(\bar{\eta}) / J_{\beta}(\bar{\eta})\right]^{2}}
$$

Here $\bar{\eta}=\eta(y=H)=\eta(\bar{y}=1)=\bar{\omega} /(1-\alpha)$. Actually the solution (25) is sufficient to find the reflection coefficient in the case of arbitrary known ratio of acoustic impedances of a substrate and granular medium at depth $H$, if necessary. In the result (26), the modulus of the reflection coefficient equals 1 as it should be for a non-absorbing material. The dependences of the phase shift on frequency $\bar{\omega}$ for $\alpha=1 / 4$ and $\alpha=1 / 6$ are presented in Figure 2. The phase shift has a simple asymptotic behavior both at low and at high frequencies

$$
\varphi \approx 2 \bar{\omega} \text { for } \quad \omega t_{H}=\bar{\omega} /(1-\alpha)<<1
$$

and

$$
\varphi \approx 2 \bar{\omega} /(1-\alpha)-(\pi / 2) \alpha /(1-\alpha) \quad \text { for } \quad \omega t_{H}=\bar{\omega} /(1-\alpha)>>1,
$$

respectively.

Here is meaningful to note the following. The sound velocity scales as $c=\gamma(\alpha) y^{\alpha}=c_{H}(y / H)^{\alpha}$, where $\gamma(\alpha)$ is depth and frequency independent. Hence for the characteristic reflection time one obtains $t_{H}=(1-\alpha)^{-1} H / c_{H}=(1-\alpha)^{-1} \gamma^{-1} H^{1-\alpha}$. Consequently, the dependences of the normalized frequency $\bar{\omega}$ and of the phases in the asymptotic expressions (27) and (28) on the layer thickness $H$ are sublinear $\left(\propto H^{1-\alpha}\right)$ and thus contain information on $\alpha$. 
So, analyzing low frequency and/or high frequency asymptotes of the phase of the reflection coefficient, it is possible to estimate the power $\alpha$ of the acoustic wave velocity depth profile in a granular medium under gravity.

The result (26) can be used for description of the reflection of signals with any spectrum. In the general case, wave profile transformation can be calculated with the help of (26) and the numerical Fourier transform. The phase shift in the formula (26) increases with increasing frequency, which means that higher frequencies approach closer to the surface. Correspondingly they spend more time in the region $0 \leq y \leq H$ than the low frequency waves. In other words, the acoustic waves of all frequencies are reflected at each point of the inhomogeneous velocity profile, but low frequency waves are efficiently reflected by smaller gradients of the velocity that is at larger distances from the surface. They will be reflected in average "earlier" than the high frequency waves.

When the phase shift is proportional to frequency the acoustic waves are reflected without their profile transformation. In accordance with the asymptote (27), if the incident acoustic pulse $u_{i}(y=H, t)=f(t)$ contains only low-frequency spectral components $\left(\omega<<(1-\alpha) c_{H} / H=t_{H}^{-1}\right)$, then the profile of the reflected wave will be $u_{r}(y=H, t)=f\left(t-2(1-\alpha) t_{H}\right)$. It can be concluded that it takes less time for the low frequencies to be reflected by the layer than for the high frequencies $\left(\omega>>(1-\alpha) c_{H} / H=t_{H}^{-1}\right)$. From the physics point of view the lower is its frequency the more sensitive is the wave to the rigidity gradients. Waves with frequencies $\omega<<t_{H}^{-1}$ are reflected along the path of their propagation, while waves with frequencies $\omega>>t_{H}^{-1}$ (rays) are reflected only at the surface. The first part of the phase $\varphi$ for the high frequency waves in the asymptote (28) is clearly related to 
the time necessary for an acoustic ray to cross twice the layer $0 \leq y \leq H$. The second part of the equation (28)

$$
\Delta \varphi_{0} \equiv-(\pi / 2) \alpha /(1-\alpha)
$$

is the so-called phase loss accumulated by the wave in reflection from the discontinuity of the elastic modulus gradient exactly at the surface [46,48]. Only for $\alpha=1 / 2$ this phase loss is equal to the one accumulated by the acoustic ray in reflection from a simple caustic $[45,46,48]$.

From the same continuity conditions of displacements and stresses at $y=H$ it is possible to find the amplitude of surface displacement caused by the incident acoustic wave

$$
\frac{\tilde{u}(0)}{\tilde{u}_{i}}=\frac{\bar{\eta}^{\beta}}{2^{\beta-1} \Gamma(\beta+1)\left[J_{\beta}(\bar{\eta})+i J_{\beta+1}(\bar{\eta})\right]}=\left|\frac{\tilde{u}(0)}{\tilde{u}_{i}}\right| e^{-i \varphi(0)},
$$

where

$$
\left|\frac{\tilde{u}(0)}{\tilde{u}_{i}}\right|=\frac{\bar{\eta}^{\beta}}{2^{\beta-1} \Gamma(\beta+1)\left[J_{\beta}^{2}(\bar{\eta})+J_{\beta+1}^{2}(\bar{\eta})\right]^{1 / 2}},
$$

and

$$
\tan \varphi(0)=\frac{J_{\beta+1}(\bar{\eta})}{J_{\beta}(\bar{\eta})}
$$

The normalized amplitude $\left|\tilde{u}(0) / \tilde{u}_{i}\right|$ has local extrema at frequencies defined by the condition $J_{\beta+1}(\bar{\eta})=J_{\beta+1}(\bar{\omega} /(1-\alpha))=0$. The existence of these extrema is due to interference of multiply reflected acoustic waves inside the layer $0 \leq y \leq H$. It is important that the exact solution (25) of 
equation (23) takes into account scattering of the forward and backward (up and down) propagating acoustic waves at each point of the volume and not only at the free surface. The dependence (30) of the surface displacement amplitude on frequency is presented in Figure 3. The high frequency $(\bar{\omega} /(1-\alpha)>>1)$ extrema are located at $\bar{\omega}_{n} \cong \pi[(1-\alpha) n+\alpha / 4]$ as it follows from the asymptotic behavior of Bessel functions [47]. Here $n>>1$ is an integer number.

At low frequencies $\left(\bar{\omega}<<t_{H}^{-1}\right)$ the surface displacement is approximately twice larger than the displacement in the incident wave $\left(\tilde{u}(0) \cong 2\left[1+\alpha(1-\alpha)\left(\omega t_{H}\right)^{2}\right] \tilde{u}_{i}\right)$ similarly to the case of acoustic wave incidence on the surface of a homogeneous half space. It means that at extremely low frequencies the incident wave does not distinguish if the layer is homogeneous or not. At high frequencies $\bar{\omega}>>t_{H}^{-1}$ the amplitude of the surface displacement slowly grows with frequency and layer thickness: $\left|\tilde{u}(0) / \tilde{u}_{i}\right| \cong 2 \sqrt{\pi}\left(\omega t_{H} / 2\right)^{\alpha /[2(1-\alpha)]} \propto \omega^{\alpha /[2(1-\alpha)]} H^{\alpha / 2}$. It looks like the wave field becomes stronger localized near the surface with increasing frequency of the incident wave. Note that both low frequency and high frequency asymptotes of the surface displacement dependence on frequency and layer thickness carry information on the parameter $\alpha$ of the depth rigidity profile.

The spatial distribution of the acoustic field from the solutions (25) and (30) $\left|\frac{\tilde{u}\left(y, \bar{\eta}=\omega t_{H}\right)}{\tilde{u}_{i}}\right|=\frac{2 \bar{y}^{1 / 2-\alpha}\left|J_{\beta}\left(\bar{\eta}^{1-\alpha}\right)\right|}{\left[J_{\beta}^{2}(\bar{\eta})+J_{\beta+1}^{2}(\bar{\eta})\right]^{1 / 2}}$ inside the granular layer $(0 \leq \bar{y} \leq 1)$ for different frequencies is shown in Figure 4. The inset in Figure 4 demonstrates that increasing acoustic frequency results in the increase of the surface displacement amplitude and in stronger spatial confinement of the acoustic field in the vicinity of the surface. This result indicates that high frequency waves could be of particular importance for the diagnostics of granular surfaces. 
Using the solution (25) it is also possible to find the resonance frequencies of the standing acoustic oscillations between the mechanically free surface at $y=0$ and absolutely rigid emitting transducer at $y=H$. They are given by the roots of the equation

$$
J_{\beta}(\bar{\eta})=J_{\beta}\left(\omega t_{H}\right)=0
$$

that yields a simple equidistant sequence $\bar{\omega}_{n} \cong \pi[(1-\alpha)(n-1 / 2)+\alpha / 4]$ for high resonant frequencies. For the derivation of the resonance condition (31) it has been assumed that there is no slip of the particles along the surface of the transducer in the case of shear resonance excitation. More complicated boundary conditions at the surface $y=0$ can be accounted in the framework of the general solution (24). In particular, it is possible to analyze the role of the adhesion between the grains in the absence of external or gravity-induced pressure through modeling of the velocity depth profiles by $c=c_{0}\left(1+y / y_{0}\right)^{\alpha}[30,31]$.

Before starting the nonlinear phenomena analysis we remind to the reader the role of the phase loss terms (of the type $\Delta \varphi_{0} \equiv-(\pi / 2) \alpha /(1-\alpha)$, see the result (29)) in the reflection of the acoustic waves. Phase loss is the only factor influencing the profiles of the reflected acoustic waves (rays) in the geometrical acoustics (high frequency) approximation. In accordance with the results (26) and (29) the reflection of the incident acoustic wave by the discontinuity of rigidity gradient at the surface is described by

$$
R(\omega>0, y=0, \alpha) \equiv \frac{\tilde{u}_{r}(0)}{\tilde{u}_{i}(0)}=\exp \left(-i \Delta \varphi_{0}\right)=\exp \left[i \frac{\pi}{2} \frac{\alpha}{(1-\alpha)}\right]
$$


When applying the above expression (32) to predict the transformation of the wave profile in reflection, it is necessary to take into account that reflection coefficient at negative frequencies can be found from formula (32) using the operation of complex conjugation $R(-\omega)=R^{*}(\omega)$. Then the application of the Fourier transforms leads to the following relation between the profiles of the reflected and incident waves:

$$
u_{r}(t, y=0)=\cos \left(\Delta \varphi_{0}\right) u_{i}(t, y=0)+\sin \left(\Delta \varphi_{0}\right) H\left[u_{i}(t, y=0)\right]
$$

where $H[\cdot]$ denotes the operator of the Hilbert transform [49]. The first term in the formula (33) corresponds to the simple reflection from the mechanically free surface of a homogeneous material. This component of the reflected wave has the same profile as the incident one and is only reduced by the factor $\cos \left(\Delta \varphi_{0}\right)$. In contrast, the second term in the solution (33) describes profile transformations and formally corresponds to the reflection from a simple caustic, where phase loss equals $-\pi / 2[36,48]$, with a reduction factor $\sin \left(\Delta \varphi_{0}\right)$ In this sense, reflection from the mechanically free surface of an unconsolidated granular material under gravity differs both from the simple caustic and from the surface of a homogeneous material.

\section{Reflection of weakly nonlinear acoustic waves}

The natural way to start the analysis of the nonlinear phenomena is to apply the method of successive approximations. We start with the analysis of the second harmonic generation in reflection of the longitudinal acoustic wave from a mechanically free surface of an unconsolidated granular medium. In addition to normalizations and assumptions adopted in the derivation of the equation (23) the mechanical displacement is normalized to its value $u_{y i}^{\prime}$ in the 
incident wave at depth $y=H$ and normalized time $\theta=\omega t$ is introduced. Then the equation (11) takes the form

$$
\frac{\partial}{\partial \bar{y}}\left[\bar{y}^{2 \alpha} \frac{\partial u}{\partial \bar{y}}\right]-\bar{\omega}^{2} \frac{\partial^{2} u}{\partial \theta^{2}}=N \frac{\partial}{\partial \bar{y}}\left[\bar{y}^{4 \alpha-1}\left(\frac{\partial u}{\partial \bar{y}}\right)^{2}\right]
$$

The non-dimensional nonlinear parameter $N$ in the equation (34) has the form $N=\alpha c_{H}^{2} u_{y i}^{\prime} /\left(g H^{2}\right)=\alpha\left(a_{y i} / g\right) / \varpi^{2}=\alpha\left|\sigma_{i}^{\prime} / \sigma_{0}\right| /\left(k_{H} H\right)$, where $a_{y i}$ and $\sigma_{i}^{\prime}$ denote particle acceleration and stress of the incident acoustic wave, respectively. For $N<<1$ the solution of the equation (34) can be found by successive approximations in the form $u=\operatorname{Re}\left\{\mu \tilde{u}_{1} \exp (i \theta)+\mu^{2}\left[\tilde{u}_{22} \exp (2 i \theta)+u_{20}\right]+\ldots\right\}$, where small non-dimensional parameter $\mu<<1$ indicates that each successive term of this expansion is much smaller than the previous. Validity of the solution obtained by successive approximations can always be achieved by diminishing the amplitude of the incident wave, and the formal mathematical condition can be also always explicitly written when necessary. Equating in (34) the terms of the first order in $\mu$ we get for $u_{1}$ the equation (23) with the corresponding solutions (25) and (30)

$$
\tilde{u}_{1}=2^{\beta} \Gamma(\beta+1)\left|\frac{\tilde{u}(0)}{\tilde{u}_{i}}\right| e^{-i \varphi(0)} \eta^{-\beta} J_{\beta}(\eta) \equiv u_{10} e^{-i \varphi(0)} \eta^{-\beta} J_{\beta}(\eta) \equiv \tilde{u}_{10} e^{-i \varphi(0)},
$$


where $\tilde{u}_{10}$ is a real function. The terms of the order $\mu^{2}$ in the equation (34) describe the generation of the second harmonic and of the average field. The second harmonic can be presented as $u_{2}=\operatorname{Re}\left\{\mu^{2} \widetilde{u}_{2} \exp (2 i \theta-2 i \varphi(0))\right\}$ and its generation is controlled by the equation

$$
\frac{\partial}{\partial \bar{y}}\left[\bar{y}^{2 \alpha} \frac{\partial \tilde{u}_{2}}{\partial \bar{y}}\right]+(2 \bar{\omega})^{2} \widetilde{u}_{2}=\frac{N}{2} \frac{\partial}{\partial \bar{y}}\left[\bar{y}^{4 \alpha-1}\left(\frac{\partial \tilde{u}_{10}}{\partial \bar{y}}\right)^{2}\right] .
$$

Searching the solution of equation (36) in the form

$$
\tilde{u}_{2}=\eta^{-\beta} \phi\left(\eta^{\prime}\right)
$$

with $\eta^{\prime}=2 \eta$, we transform equation (36) into inhomogeneous Bessel equation

$$
\begin{aligned}
\eta^{\prime 2} \frac{\partial^{2} \phi}{\partial \eta^{\prime 2}}+\eta^{\prime} \frac{\partial \phi}{\partial \eta^{\prime}}+\left(\eta^{\prime 2}-\beta^{2}\right) \phi & =\frac{N}{2} 2^{2 \beta} \frac{u_{10}^{2} \bar{\omega}^{2}}{(1-\alpha)} \eta^{\prime-\beta} \frac{\partial}{\partial \eta^{\prime}}\left[J_{\beta+1}^{2}\left(\frac{\eta^{\prime}}{2}\right)\right] \\
& \equiv N^{\prime} \eta^{\prime 1-\beta} \frac{\partial}{\partial \eta^{\prime}}\left[J_{\beta+1}^{2}\left(\frac{\eta^{\prime}}{2}\right)\right] \equiv N^{\prime} g\left(\eta^{\prime}\right)
\end{aligned}
$$

Since the parameter $\beta$ in the left-hand-side of equation (38) is not an integer for the typical values of $\alpha$ of interest here, it is possible and suitable to chose for two linearly independent solutions of the homogeneous equation the Bessel functions of the first kind $\phi_{1}=J_{\beta}\left(\eta^{\prime}\right)$ and $\phi_{2}=J_{-\beta}\left(\eta^{\prime}\right)$. Then the general solution of the inhomogeneous equation (38) [50] 
$\phi=c_{1} \phi_{1}+c_{2} \phi_{2}+\phi_{2} \int \phi_{1} \frac{g}{h} \frac{d \eta^{\prime}}{W}-\phi_{1} \int \phi_{2} \frac{g}{h} \frac{d \eta^{\prime}}{W}$, where $W=\phi_{1} \phi_{2}^{\prime}-\phi_{2} \phi_{1}^{\prime}$ and $h=\eta^{\prime 2}$ is the coefficient in front of the second derivative in the equation (34), can be rewritten in the form

$$
\begin{aligned}
& f=c_{1} J_{\beta}\left(\eta^{\prime}\right)+c_{2} J_{-\beta}\left(\eta^{\prime}\right)-N^{\prime} \frac{\pi}{2 \sin (\beta \pi)} \times \\
& \times\left\{J_{\beta}\left(\eta^{\prime}\right) \int J_{\beta+1}^{2}\left(\frac{\eta^{\prime}}{2}\right) J_{-(\beta+1)}\left(\eta^{\prime}\right) \frac{d \eta^{\prime}}{\eta^{\prime \beta}}+J_{-\beta}\left(\eta^{\prime}\right) \int J_{\beta+1}^{2}\left(\frac{\eta^{\prime}}{2}\right) J_{\beta+1}\left(\eta^{\prime}\right) \frac{d \eta^{\prime}}{\eta^{\prime \beta}}\right\}
\end{aligned}
$$

To arrive to this presentation we have used the integration by parts and the Wronskian of the Bessel functions $W=J_{\beta} J_{-\beta}^{\prime}-J_{-\beta} J_{\beta}^{\prime}=J_{\beta+1} J_{-\beta}+J_{-(\beta+1)} J_{\beta}=-2 \sin (\beta \pi) /\left(\pi \eta^{\prime}\right)$ [47]. Finally, when substituting the obtained solution for $f$ in the form (37), the limits of integration should be fixed together with the constant $c_{2}$ to satisfy stress-free boundary conditions at the surface $\eta^{\prime}=0$. A rather suitable form of presentation of the solution for the second harmonic is

$$
\begin{aligned}
& \tilde{u}_{2}=2^{\beta} \Gamma(\beta+1) \tilde{u}_{2}(0) \frac{J_{\beta}\left(\eta^{\prime}\right)}{\eta^{\prime \beta}}- \\
& -\frac{\pi N^{\prime} \eta^{\prime-\beta}}{2 \sin (\beta \pi)}\left\{J_{\beta}\left(\eta^{\prime}\right) \int_{0}^{\eta^{\prime}} J_{\beta+1}^{2}\left(\frac{\eta^{\prime \prime}}{2}\right) J_{-(\beta+1)}\left(\eta^{\prime \prime}\right) \frac{d \eta^{\prime \prime}}{\eta^{\prime \prime}}+J_{-\beta}\left(\eta^{\prime}\right) \int_{0}^{\eta^{\prime}} J_{\beta+1}^{2}\left(\frac{\eta^{\prime \prime}}{2}\right) J_{\beta+1}\left(\eta^{\prime \prime}\right) \frac{d \eta^{\prime \prime}}{\eta^{\prime \prime}}\right\}
\end{aligned}
$$

In the solution (39) $\tilde{u}_{2}(0)$ denotes the spectral amplitude of the second harmonic at the surface. To find this surface displacement and to find the second harmonic emitted from the boundary $y=H$ into the acoustically matched substrate (Figure 1), it should be taken into account that there is no field of the second harmonic incident from the substrate at the boundary $y=H$ and, correspondingly, the second harmonic acoustic field at $y=H+0$ can be presented as an emitted 
(reflected) wave only $\tilde{u}_{2}(y=H+0)=\tilde{u}_{2 r} \exp \left[-2 i k_{H}(y-H)\right]$. The continuity conditions for the displacement and stress at $y=H$, when this solution is matched to the solution (39), provide two equations to find the surface displacement $\tilde{u}_{2}(0)$ and the displacement of the reflected (emitted) second harmonic $\tilde{u}_{2 r}$. The final results for the second harmonic at the mechanically free surface and for the harmonics reflected from the granular layer can be presented in the form

$$
\begin{gathered}
\frac{\tilde{u}_{2}(0)}{N \bar{\omega}^{2}}=-\frac{1}{(1-\alpha) \Gamma(\beta+1)} \frac{\bar{\eta}^{\beta-1}}{J_{\beta+1}^{2}(\bar{\eta})+J_{\beta}^{2}(\bar{\eta})}\left\{\frac{\pi(2 \bar{\eta})^{(\beta+1)}}{2 \sin (\beta \pi)}\left[\frac{J_{\beta+1}(2 \bar{\eta}) J_{-(\beta+1)}(2 \bar{\eta})-J_{\beta}(2 \bar{\eta}) J_{-\beta}(2 \bar{\eta})}{J_{\beta+1}^{2}(2 \bar{\eta})+J_{\beta}^{2}(2 \bar{\eta})} I_{+}(2 \bar{\eta})-I_{-}(2 \bar{\eta})\right]\right. \\
\left.-\frac{J_{\beta+1}^{2}(\bar{\eta}) J_{\beta+1}(2 \bar{\eta})}{J_{\beta+1}^{2}(2 \bar{\eta})+J_{\beta}^{2}(2 \bar{\eta})}-i \frac{(2 \bar{\eta})^{\beta} I_{+}(2 \bar{\eta})+J_{\beta+1}^{2}(\bar{\eta}) J_{\beta}(2 \bar{\eta})}{J_{\beta+1}^{2}(2 \bar{\eta})+J_{\beta}^{2}(2 \bar{\eta})}\right\} \cdot \\
\frac{\tilde{u}_{2 r}=\left|\tilde{u}_{2 r}\right| e^{-i \varphi_{2 r}}}{N \bar{\omega}^{2}}=\frac{2^{\beta}}{(1-\alpha)} \frac{(\bar{\eta})^{\beta-1}}{\left[J_{\beta+1}^{2}(\bar{\eta})+J_{\beta}^{2}(\bar{\eta})\right]} \frac{\left[(2 \bar{\eta})^{-\beta} J_{\beta}(2 \bar{\eta}) J_{\beta+1}^{2}(\bar{\eta})+I_{+}(2 \bar{\eta})\right]}{\left[J_{\beta+1}^{2}(2 \bar{\eta})+J_{\beta}^{2}(2 \bar{\eta})\right]^{1 / 2}} \\
\tan \varphi_{2 r}=\frac{J_{\beta}(2 \bar{\eta})}{J_{\beta+1}(2 \bar{\eta})}
\end{gathered}
$$

where the compact notation $I_{ \pm}$are introduced for the integrals $I_{ \pm}(x) \equiv \int_{0}^{x} J_{\beta+1}^{2}\left(\frac{x^{\prime}}{2}\right) J_{ \pm(\beta+1)}\left(x^{\prime}\right) \frac{d x^{\prime}}{x^{\prime \beta}}$.

The dependences of the displacement amplitudes $\left|\tilde{u}_{2}(0)\right|$ and $\left|\tilde{u}_{2 r}\right|$ on the normalized thickness of the granular layer are presented in Figure 5 and Figure 6, respectively. These results will be discussed later in Section 5 together with similar dependences for the third harmonic excited in the reflection of a shear acoustic wave.

We are turning to the analysis of the nonlinear reflection of shear acoustic waves. When we retain all the normalizations proposed before for the derivation of equation (34), but 
normalize the displacement on its value $u_{x i}^{\prime}$ in the incident wave at depth $y=H$, the equation (21) for the shear waves in quadratic approximation of the nonlinear acoustics transforms into:

$\frac{\partial}{\partial \bar{y}}\left[\bar{y}^{2 \alpha} \frac{\partial u}{\partial \bar{y}}\right]-\bar{\omega}^{2} \frac{\partial^{2} u}{\partial \theta^{2}}=\frac{N}{f} \frac{\partial}{\partial \bar{y}}\left\{\bar{y}^{4 \alpha-1}\left[\left(\frac{\partial u}{\partial \bar{y}}\right)_{\max } \frac{\partial u}{\partial \bar{y}}-\frac{1}{2}\left(\left(\frac{\partial u}{\partial \bar{y}}\right)_{\max }^{2}-\left(\frac{\partial u}{\partial \bar{y}}\right)^{2}\right) \operatorname{sign}\left(\frac{\partial^{2} u}{\partial \bar{y} \partial \theta}\right)\right]\right\} .(42$

The solution of equation (42) can be found by the method of successive approximations in the form $u=\operatorname{Re}\left\{\mu \tilde{u}_{1} \exp (i \theta)+\mu^{2}\left[\tilde{u}_{21} \exp (i \theta)+\tilde{u}_{23} \exp (3 i \theta)+\tilde{u}_{25} \exp (5 i \theta)+\ldots\right]+\ldots\right\}$. The solution of the linearized equation at the first step of successive approximations coincides with the solution (35) for the longitudinal waves. In the second order of successive approximations in media with hysteretic quadratic nonlinearity, all the odd harmonics are generated simultaneously and there is also self-action at the fundamental frequency [51]. The first term in the square bracket in the equation (42) contributes to the signal at fundamental frequency only. The second term contributes to all odd harmonics and to the fundamental frequency as well.

We present below only the results for the third harmonic. The predictions for the other spectral components can be obtained similarly if it is necessary for the analysis of the experimental data. Presenting the third harmonic field in the form $u_{3}=\operatorname{Re}\left\{\mu^{2} \tilde{u}_{3} \exp (3 i \theta-3 i \varphi(0)+i \pi / 2)\right\}$ for the complex spectral amplitude $\tilde{u}_{3}$ we derive the equation

$$
\frac{\partial}{\partial \bar{y}}\left[\bar{y}^{2 \alpha} \frac{\partial \tilde{u}_{3}}{\partial \bar{y}}\right]+(3 \bar{\omega})^{2} \tilde{u}_{3}=\frac{4}{15 \pi} \frac{N}{f} \frac{\partial}{\partial \bar{y}}\left[\bar{y}^{4 \alpha-1}\left(\frac{\partial \tilde{u}_{10}}{\partial \bar{y}}\right)^{2} \operatorname{sign}\left(\frac{\partial \tilde{u}_{10}}{\partial \bar{y}}\right)\right]
$$

The most important qualitative difference of the equation (43) for the third harmonic in comparison with the equation (36) for the second harmonic is the additional multiplier 
$\operatorname{sign}\left(\partial \tilde{u}_{10} / \partial \bar{y}\right)$ in the right-hand-side, which changes the symmetry of the nonlinearity from an even type to that of an odd type. Searching the solution of (43) in the form $\tilde{u}_{3}=\eta^{\prime-\beta} \phi\left(\eta^{\prime}\right)$ with $\eta^{\prime}=3 \eta$, we transform the equation (43) into inhomogeneous Bessel equation

$$
\begin{aligned}
& \eta^{\prime 2} \frac{\partial^{2} \phi}{\partial \eta^{\prime 2}}+\eta^{\prime} \frac{\partial \phi}{\partial \eta^{\prime}}+\left(\eta^{\prime 2}-\beta^{2}\right) \phi=-\frac{64 N}{21} 3^{\beta} \frac{\tilde{u}_{10}^{2} \bar{\omega}^{2}}{(1-\alpha)} \eta^{\prime 1-\beta} \frac{\partial}{\partial \eta^{\prime}}\left[J_{\beta+1}\left(\frac{\eta^{\prime}}{2}\right)\left|J_{\beta+1}\left(\frac{\eta^{\prime}}{2}\right)\right|\right], \\
& \equiv N^{\prime} \eta^{\prime 1-\beta} \frac{\partial}{\partial \eta^{\prime}}\left[J_{\beta+1}\left(\frac{\eta^{\prime}}{2}\right)\left|J_{\beta+1}\left(\frac{\eta^{\prime}}{2}\right)\right|\right] \equiv N^{\prime} g\left(\eta^{\prime}\right)
\end{aligned}
$$

where $N^{\prime}$ and $g\left(\eta^{\prime}\right)$ differ from those introduced in the equation (38). Otherwise the solution of equation (44) is absolutely the same as one obtained earlier for the second harmonics. For example, the solution for $\tilde{u}_{3}$ can be obtained by he following substitutions in the solution (39): $\tilde{u}_{2} \Rightarrow \tilde{u}_{3}, \quad \tilde{u}_{2}(0) \Rightarrow \tilde{u}_{3}(0)$, and $J_{\beta+1}^{2}\left(\eta^{\prime} / 2\right) \Rightarrow J_{\beta+1}\left(\eta^{\prime} / 3\right)\left|J_{\beta+1}\left(\eta^{\prime} / 3\right)\right|$. It should be also taken into account that $N^{\prime}$ in the equation (44) differs by a factor $-(128 / 21)(3 / 2)^{\beta}$ from $N^{\prime}$ in the equation (38). With all necessary substitutions the solutions of the equation (43) are presented in the following form:

$$
\begin{aligned}
f \frac{\tilde{u}_{3}(0)}{N \bar{\omega}^{2}} & =-\frac{16}{21} \frac{3^{\beta}}{\Gamma(1-\alpha)} \frac{\bar{\eta}^{\beta-1}}{J_{\beta+1}^{2}(\bar{\eta})+J_{\beta}^{2}(\bar{\eta})}\left\{\frac{\pi(3 \bar{\eta})^{(\beta+1)}}{2 \sin (\beta \pi)}\left[\frac{J_{\beta+1}(3 \bar{\eta}) J_{-(\beta+1)}(3 \bar{\eta})-J_{\beta}(3 \bar{\eta}) J_{-\beta}(3 \bar{\eta})}{J_{\beta+1}^{2}(3 \bar{\eta})+J_{\beta}^{2}(3 \bar{\eta})} I_{+}(3 \bar{\eta})-I_{-}(3 \bar{\eta})\right]\right. \\
& \left.-\frac{J_{\beta+1}(\bar{\eta})\left|J_{\beta+1}(\bar{\eta})\right| J_{\beta+1}(3 \bar{\eta})}{J_{\beta+1}^{2}(3 \bar{\eta})+J_{\beta}^{2}(3 \bar{\eta})}-i \frac{(3 \bar{\eta})^{\beta} I_{+}(3 \bar{\eta})+J_{\beta+1}(\bar{\eta})\left|J_{\beta+1}(\bar{\eta})\right| J_{\beta}(3 \bar{\eta})}{J_{\beta+1}^{2}(3 \bar{\eta})+J_{\beta}^{2}(3 \bar{\eta})}\right\} .
\end{aligned}
$$

for the third harmonics at the surface and 


$$
\begin{gathered}
\tilde{u}_{3 r}=\left|\tilde{u}_{3 r}\right| e^{-i\left(\pi / 2+\varphi_{3 r}\right)}, \\
f \frac{\left|\tilde{u}_{3 r}\right|}{N \bar{\omega}^{2}}=\frac{16}{21} \frac{3^{\beta}}{(1-\alpha)} \frac{(\bar{\eta})^{\beta-1}}{\left[J_{\beta+1}^{2}(\bar{\eta})+J_{\beta}^{2}(\bar{\eta})\right]} \frac{\left|(3 \bar{\eta})^{-\beta} J_{\beta}(3 \bar{\eta}) J_{\beta+1}(\bar{\eta})\right| J_{\beta+1}(\bar{\eta})\left|+I_{+}(3 \bar{\eta})\right|}{\left[J_{\beta+1}^{2}(3 \bar{\eta})+J_{\beta}^{2}(3 \bar{\eta})\right]^{1 / 2}}, \\
\tan \varphi_{3 r}=\frac{J_{\beta}(3 \bar{\eta})}{J_{\beta+1}(3 \bar{\eta})} .
\end{gathered}
$$

for the third harmonics reflected from the granular layer. These dependences as functions of the normalized thickness of the granular layer are presented in Figure 7 and Figure 8, respectively,

\section{Discussion}

The local extrema in the dependences of the harmonics amplitudes on the layer thickness in Figures 6-8 and corresponding features in Figure 5 are related to interference (resonance) phenomena at frequencies $\omega, 2 \omega$ and $3 \omega$. All four figures demonstrate an important growth of the harmonics for increasing layer thickness up to $\bar{\eta} \leq 10$. Asymptotic expansion of the equations (40), (41), (45) and (46) for $\bar{\eta}<<1$ demonstrates that for very thin layers, where there is no significant difference in efficiency of harmonics generation by synchronous (accumulating) interaction of co-propagating waves and asynchronous interaction of counter-propagating waves, all the nonlinear quantities $\left|\tilde{u}_{N L}\right| /\left(N \bar{\omega}^{2}\right)$ presented in Figures 6-8 are linear on the normalized layer thickness $\left(\left|\tilde{u}_{N L}\right| /\left(N \bar{\omega}^{2}\right) \propto \bar{\eta}\right)$. We remind here that $\bar{\eta}=\varpi /(1-\alpha) \propto \omega H^{1-\alpha}$, while $N \bar{\omega}^{2}=\left(\alpha u_{y i}^{\prime} / g\right) \omega^{2}$ does not depend on layer thickness $H$. Consequently for $\bar{\eta} \ll<$ the dependence of all evaluated displacement amplitudes on frequency is cubic $\left(\left|\tilde{u}_{N L}\right| \propto \omega^{3}\right)$. The dependence of all the amplitudes on the layer thickness is sublinear $\left(\left|\tilde{u}_{N L}\right| \propto H^{1-\alpha}\right)$. The latter 
sublinear scaling law could be considered as one of the fingerprints of a granular medium with power-law variation of elastic properties, in comparison with homogeneous layers where under similar approximations the scaling is linear: $\left|\tilde{u}_{N L}\right| \propto H$.

The results in Figures 5-8 indicate that with increasing $|\bar{\eta}|$ (for $1 \leq|\bar{\eta}| \leq 10$ ) the scaling of the harmonic with layer thickness becomes much more sublinear (more saturated) than in the asymptotic limit $|\bar{\eta}| \ll<1$. An important feature of the results is a much faster saturation in the amplitude of the emitted second harmonic amplitude $\left|\tilde{u}_{2 r}\right|$ in comparison with other amplitudes and, in particular, in comparison with $\left|\tilde{u}_{3 r}\right|$. Qualitative explanation of this predicted phenomenon is based on the analysis of the reflection of the fundamental wave and the harmonics (generated in upward propagation of the fundamental wave) exactly at the free surface of the granular layer in the framework of the theoretical solutions (29), (32) and (33). For this purpose, we remind that in reflection from a mechanically free surface of an elastically homogeneous medium

1) displacement waves of all frequencies are reflected without a phase shift;

2) nonlinear sources created by the fundamental frequency, which are exciting the harmonics, are inverted ( $\pi$ phase shifted) in acoustic field reflection in the case of eventype nonlinearity;

3) nonlinear sources created by the fundamental frequency, which are exciting the harmonics, do not exhibit any phase shift in acoustic field reflection in the case of oddtype nonlinearity.

Known consequences of this are following. In the case of an ordinary elastic quadratic nonlinearity (even parity) for the longitudinal waves, the second harmonic generated in upward 
propagation can be completely damped (compensated) by the second harmonic generated in the downward propagation, since they are generated in antiphase. In contrast, in the case of an ordinary elastic cubic nonlinearity (odd parity) for the shear or longitudinal waves, the third harmonic will continue to accumulate along the wave propagation path even after the reflection of the acoustic field from the free surface. As an example, these phenomena make necessary to include cubic elastic nonlinearity in the analysis of the open-end gas acoustic resonators, as the quadratic elastic nonlinearity could be ineffective for the saturation of the oscillation amplitude (see $[52,53]$ and the references therein). In the case of a granular medium under gravity analyzed here, an important difference in the scale of the saturation of the reflected longitudinal second harmonic (Figure 6) and the shear third harmonic (Figure 8) could be attributed to the fact that they are generated by the nonlinearities of different parity. The elastic quadratic nonlinearity for longitudinal waves is even, while the hysteretic quadratic nonlinearity for the shear waves is odd. It should be also taken into account that for $\alpha \leq 1 / 4$ typical of longitudinal waves, the phase shift $\Delta \varphi_{0}$ (expression (29)), accumulated in reflection from infinite gradient of material rigidity exactly at the surface $(y=0)$, does not exceed $\pi / 6$. Then, in accordance with the solution (33) more than $87 \%$ of the incident wave profile is reflected without transformation as at a free surface of a homogeneous layer. Thus we attribute faster saturation of $\left|\tilde{u}_{2 r}\right|$ (Figure 6) in comparison with $\left|\tilde{u}_{3 r}\right|($ Figure 8) to the role of the phase transformations of the acoustic field in reflection from a free surface, which influence differently the phase between the reflected harmonics and the nonlinear sources in the reflected wave in case of an even-type and an oddtype nonlinearities. Note that the absence of qualitative difference in the behavior of the surface displacement amplitudes $\left|\tilde{u}_{2}(0)\right|$ (Figure 5) and $\left|\tilde{u}_{3}(0)\right|$ (Figure 7) demonstrates that there is no qualitative difference in the accumulation of longitudinal and shear harmonics in upward 
propagation of the fundamental wave. This observation confirms that the qualitative differences in the behavior of $\left|\tilde{u}_{2 r}\right|$ (Figure 6) and $\left|\tilde{u}_{3 r}\right|$ (Figure 8) are initiated by the reflection from the mechanically free surface of a granular medium.

For the intermediate regime $1 \leq \bar{\eta} \leq 40$ presented in Figures 5-8 it is difficult to provide simpler analytical (approximate) description than one in equations (40), (41), (45), (46) because of the significance of the interference phenomena for $1 \leq \bar{\eta} \leq 10$ and, more importantly, because of a very slow saturation of the integrals $I_{ \pm}$with increasing $\bar{\eta}$. The amplitude of the oscillating functions under the integrals decreases very slowly $\left(\propto 1 / x^{1+\delta(\alpha)}\right.$, with $\delta(\alpha)=\alpha /[2(1-\alpha)]$, $\delta(\alpha=1 / 4)=1 / 6, \delta(\alpha=1 / 6)=1 / 10)$. Only when the integrals are saturated the asymptotic behavior for $|\bar{\eta}| \rightarrow \infty$ can be compactly described in analytical form $\left|\tilde{u}_{N L}(0)\right| /\left(N \varpi^{2}\right) \propto \bar{\eta}^{\alpha /(1-\alpha)}$, $\left|\tilde{u}_{N L r}\right| /\left(N \varpi^{2}\right) \propto \bar{\eta}^{\alpha /[2(1-\alpha)]}$, leading to $\left|\tilde{u}_{2,3}(0)\right| \propto \omega^{(2-\alpha) /(1-\alpha)},\left|\tilde{u}_{2 r, 3 r}\right| \propto \omega^{(4-3 \alpha) /(1-\alpha)}$ and

$$
\left|\tilde{u}_{2,3}(0)\right| \propto H^{\alpha},\left|\tilde{u}_{2 r, 3 r}\right| \propto H^{\alpha / 2} .
$$

Note that the scaling laws for very long interaction distances are the same for both types (even and odd) of quadratic nonlinearity. This is another consequence of the specific reflecting properties of granular media with the power-law stratification in their mechanical properties. In accordance with the solution (33) there is always a part of the acoustic field that is reflected not in phase with the incident field. As a result the phase relations between the reflected harmonics and the nonlinear sources in the reflected field are not exactly the same as in the case of a homogeneous layer with a free surface. Because of this the complete compensation of nonlinear signals generated in upward and downward propagation of acoustic field is impossible. Even in 
the case of quadratic elastic nonlinearity, there is a continuous accumulation of the emitted harmonic with increasing thickness of the granular layer, although an extremely slow one, as it is seen in Figure 6 for $|\bar{\eta}| \geq 10$ and is predicted by the scaling (47). The fact that a free surface of a granular medium provides a phase loss intermediate between 0 and $\pi$ (corresponding to the reflection from a free and a rigid boundary of an homogeneous material, respectively) smoothes the difference between nonlinear reflection phenomena in case of even-type and odd-type nonlinearities for very thick layers. The scaling laws (47) is the second fingerprint of the nonlinear propagation in granular media with power-law distributions of linear and nonlinear properties.

The asymptotic analysis of the phases of the harmonics can be also performed with the help of the solutions (41) and (46). It indicates that the phases of the emitted harmonics $\varphi_{2 r, 3 r}$ scale linearly with the non-dimensional thickness $\bar{\eta}$ in the both asymptotic limits $\bar{\eta} \ll<$ and $\vec{\eta}>>1$. Consequently, both at low and at extremely high frequencies the phases scale linearly with $\omega$, and in both thin and very thick layers the phases scale sublinearly with layer thickness $H\left(\propto H^{1-\alpha}\right)$.

\section{Conclusions}

In this paper it is demonstrated that, due to the existence of the exact analytical solutions for the linear reflection of acoustic waves from a mechanically free surface of an unconsolidated granular medium under gravity, nonlinear reflection phenomena can be efficiently analyzed by the method of successive approximations. The developed theory revealed the differences in the nonlinear reflection process for the longitudinal and shear acoustic waves caused by the difference in parity of the nonlinearities (elastic and hysteretic, respectively) inducing these 
processes. At the same time, it is found that some specific reflection properties of a free granular surface diminish the difference between some nonlinear phenomena in longitudinal and shear waves which could be observed in reflection from a free surface of a homogeneous medium. The theory predicted specific for a granular medium with a power-law stratification of the linear and nonlinear properties dependences of the generated harmonics on frequency and on the granular layer thickness. It is obtained that the amplitudes of the generated harmonics always scale sublinearly with the increasing distance of nonlinear interaction. Theoretically obtained laws of variation of the amplitude and the phase of both linear and nonlinear acoustic signals could be used through the comparison with experimental data to evaluate the parameter $\alpha$, which characterizes the variation of the mechanical properties of unconsolidated granular media with applied pressure including the regime of low pressures in the vicinity of the jamming transition.

\section{Acknowledgements}

This study has been supported by ANR project No. NT05-341989. One of the authors (V. Aleshin) particularly acknowledges the financial support from CNRS and Pays-de-la Loire region. 


\section{References}

[1] H.M. Jager, S.R. Nagel, and R.P. Behringer: The physics of granular materials. Physics Today 49 (1996) 32-38.

[2] P.G. de Gennes: Granular matter: a tentative view. Rev. Mod. Phys. 71 (1999) S374-S382.

[3] C.-h. Liu, S.R. Nagel: Sound in a granular material: disorder and nonlinearity. Phys. Rev. B 48 (1993) 15646-15650.

[4] H.A. Makse, D.L. Johnson, L.M. Schwartz: Packing of compressible granular materials. Phys. Rev. Lett. 84 (2000) 4160-4163.

[5] C.S. O’Hern, S.A. Langer, A.J. Liu, S.R. Nagel: Random packing of frictionless particles. Phys. Rev. Lett. 88 (2002) 0755507.

[6] C.S. O’Hern, L.E. Silbert, A.J. Liu, S.R. Nagel: Jamming at zero temperature and zero applied stress: the epitome of disorder. Phys. Rev. E 68 (2003) 011306.

[7] W.G. Ellenbroek, E. Somfai, M. van Hecke, W. van Saarloss: Critical scaling in linear response of frictionless granular packings near jamming. Phys. Rev. Lett. 97 (2006) 258001.

[8] E. Somfai, M. van Hecke, W.G. Ellenbroek, K. Shundyak, W. van Saarloos: Critical and noncritical jamming of frictional grains. Phys. Rev. E 75 (2007) 020301(R).

[9] K. Shundyak, M. van Hecke, W. van Saarloos: Force mobilization and generalized isostaticity in jammed packings of frictional grains. Phys. Rev. E 75 (2007) 010301(R).

[10] T.S. Hajmudar, M. Sperl, S. Luding, R.P. Behringer: Jamming transition in granular systems. Phys. Rev. Lett. 98 (2007) 058001.

[11] J.E. White: Underground sound. Elsevier, Amsterdam, 1983.

[12] R.D. Stoll: Sediment acoustics. Springel-Verlag, Berlin, 1989.

[13] V. Tournat, V.E. Gusev and B. Castagnède: Chapter 2, Evaluation non destructive des solides micro-inhomogènes par des méthodes acoustiques non linéaires (in French), Matériaux et 
acoustique 3: caractérisation non destructive et application médicale (Matériaux et Acoustique, Traité MIM, série alliages métalliques), M. Bruneau and C. Potel coordinators, Hermès, Paris, 2006.

[14] N.A. Peterson: Seismic wave propagation in porous granular media. Geophysics 21 (1956) 691-714.

[15] B.O. Hardin, F.E. Richart: Elastic wave velocities in granular soils. Journal of Soil Mechanics and Foundations Division, ASCE (February 1963) 33-65.

[16] B.O. Hardin, W.L. Black: Sand stiffness under various triaxial stresses. Journal of Soil Mechanics and Foundations Division, ASCE (March 1966) 27-42.

[17] S.N. Domenico: Elastic properties of unconsolidated porous sand reservoirs. Geophysics $\mathbf{4 2}$ (1977) 1339-1368.

[18] H.A. Makse, N. Gland, D.L. Johnson, L. Schwartz: Granular packings nonlinear elasticity, sound propagation, and collective relaxation dynamics. Phys. Rev. E 70 (2004) 061302.

[19] L.V. Zarembo, V.A. Krasilnikov: Nonlinear phenomena in the propagation of elastic waves in solids. Sov. Phys. Uspekhi 13 (1971) 778-797.

[20] Y. Zheng, R. Maev, I.Y. Solodov: Nonlinear acoustic applications for materials characterization. Can. J. Phys. 77 (1999) 927-967.

[21] I.Yu. Belyaeva, V.Yu. Zaitsev, E.M. Timanin: Experimental study of nonlinear elastic properties of granular media with nonlinear packing. Acoust. Phys. 40 (1994) 789-793.

[22] V. Tournat, V.E. Gusev, V.Yu. Zaitsev and B. Castagnède: Acoustic second harmonic generation with shear to longitudinal mode conversion in granular media. Europhys. Lett. 66 (6) (2004) 798-804.

[23] V.Yu. Zaitsev, A.B. Kolpakov, V.E. Nazarov: Detection of acoustic pulses in river sand: Experiment. Acoust. Phys. 45 (1999) 235-241. 
[24] V. Tournat, V. Zaitsev, V. Gusev, V. Nazarov, P. Béquin, B. Castagnède: Probing weak forces in granular media through nonlinear dynamic dilatancy: clapping contacts and polarization anisotropy. Phys. Rev. Lett. 92 (2004) 085502.

[25] A. Moussatov, B. Castagnède, and V.E. Gusev: Observation of nonlinear interaction of acoustic waves in granular materials: demodulation process. Phys. Lett. A 283 (2001) 216-223.

[26] V. Tournat, V.E. Gusev, B. Castagnède: Subharmonics and noise excitation in transmission of acoustic waves through unconsolidated granular medium. Phys. Lett. A 326 (2004) 340-348.

[27] V.Yu. Zaitsev, V.E. Nazarov, V. Tournat, V. Gusev and B. Castagnède: Luxembourg-Gorky effect in a granular medium: probing perturbations of the material state via cross-modulation of elastic waves. Europhys. Lett. 70 (5) (2005) 607-613.

[28] B. Andreotti: The song of dunes as a wave-particle mode locking. Phys. Rev. Lett. 93 (2004) 238001.

[29] P. Leclaire, V. Tournat, O. Dazel, V. Aleshin, J.-F. Allard, W. Lauriks and V. Gusev: Waveguide acoustic modes for testing surface layers of unconsolidated granular media. 4th International Conference on Emerging Technologies in NDT, Stuttgart, Germany (2007), accepted.

[30] V.E. Gusev, V. Aleshin, V. Tournat: Acoustic waves in an elastic channel near the free surface of granular media. Phys. Rev. Lett. 96 (2006) 214301.

[31] V. Aleshin, V. Gusev, V. Tournat: Acoustic modes propagating along the free surface of granular media. J. Acoust. Soc. Am. (2007), accepted.

[32] F. Gassmann: Elastic waves through a packing of spheres. Geophysics 16 (1951) 673-685.

[33] J.D. Goddard: Nonlinear elasticity and pressure dependent wave speed in granular media. Proc. R. Soc. London, Ser. A 430 (1990) 105-131. 
[34] B. Velicky, C. Caroli: Pressure dependence of the sound velocity in a two-dimensional lattice of Hertz-Mindlin balls: Mean-field description. Phys. Rev. E 65 (2002) 021307.

[35] A.N. Bogdanov, A.T. Skvortsov: Nonlinear shaer waves in granular medium. Sov. Phys. Acoust. 38 (1992) 224-226.

[36] L.A. Ostrovsii, E.N. Pelinovskii, V.E. Fridman: propagation of finite amplitude sound waves in an inhomogeneous medium with caustics. Sov. Phys. Acoust. 22 (1976) 516-520.

[37] O.V. Rudenko, S.I. Soluyan: Theoretical formulations of nonlinear acoustics. Consultants Bureau, New York, 1978.

[38] V.E. Gusev, V. Aleshin and V. Tournat: Réflection en incidence normale d'ondes acoustiques non linéaires par la surface libre horizontale d'un milieu granulaire non consolidé (in French). Congrès Français d'Acoustique, Tours, France (2006).

[39] K.L. Johnson: Contact mechanics, Cambridge University Press, Cambridge, 1985.

[40] R.D. Mindlin, H. Deresiewicz: Elastic spheres in contact under varying oblique forces. J. Appl. Mech. (September 1953) 327-344.

[41] Y. Jiang, H. Liu: Granular elasticity without the Coulomb condition. Phys Rev. Lett. 91 (2003) 144301.

[42] Y. Jiang, H. Liu: Energetic instability unjams sand and suspension. Phys. Rev. Lett. 93 (2004) 148001.

[43] V.V. Krylov: New type of vibration dampers utilising the effect of acoustic 'black holes'. Acustica Acta Acustica 90 (2004) 830-837.

[44] M.A. Mironov: Propagation of a flexural wave in a plate whose thickness decreases smoothly to zero in a finite interval. Sov. Phys. Acoust. 34 (1988) 318-319; Akust. Zh. 34 (1988) $546-547$. 
[45] O.A. Godin D.M.F. Chapman: Shear-speed gradients and ocean seismo-acoustic noise resonances. J. Acoust. Soc. Am. 106 (1999) 2367-2382.

[46] O.A. Godin and D.M.F. Chapman: Dispersion of interface waves in sediments with powerlaw shear speed profiles. I. Exact and approximate analytical results. J. Acoust. Soc. Am. 110 (2001) 1890-1907.

[47] Handbook of Mathematical Functions with Formulas, Graphs, and Tables, Appl. Math. Ser., Vol. 55, edited by M. Abramovitz and I.A. Stegun (National Bureau of Standards, Washington, 1964).

[48] L.M. Brekhovskikh, O.A. Godin: Acoustics of layered media I: Plane and quasi-plane waves. Springer-Verlag, Berlin, 1990.

[49] Tables of integral transforms, based on notes left by H. Bateman, Volume II. McGraw-Hill, New York, 1954.

[50] A.D. Polyanin, V.F. Zaitsev: Handbook of exact solutions for ordinary differential equations. CRC Press, Boca Raton, 1995.

[51] V. Gusev, C. Glorieux, W. Lawriks, J. Thoen: Nonlinear bulk and surface shear acoustic waves in materials with hysteresis and end-points memory. Phys. Lett. A 232 (1997) 77-86.

[52] M.A. Ilgamov, R.B. Zaripov, R.G. Galiullin, V.B. Repin: Nonlinear oscillations of a gas in a tube. Applied Mechanics Reviews 46 (1996) 137-154.

[53] W. Chester: Resonant oscillations of a gas in an open-ended tube. Proc. R. Soc. Lond. A 377 (1981) 449-467. 


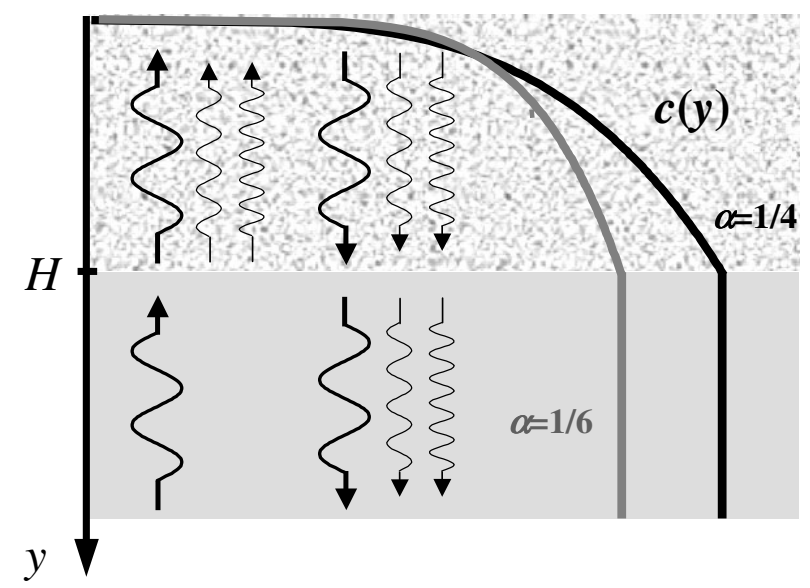

\section{Figure 1}

Reflection of plane acoustic waves from a layer of a granular material of thickness $H$. Axis $y$ of the coordinate system is along the gravity direction $\vec{g}$. Assumed depth profiles of sound velocity $c$ are presented for two power-law dependences on pressure $p\left(c(p) \propto p^{\alpha}, \alpha=1 / 6,1 / 4\right)$. 


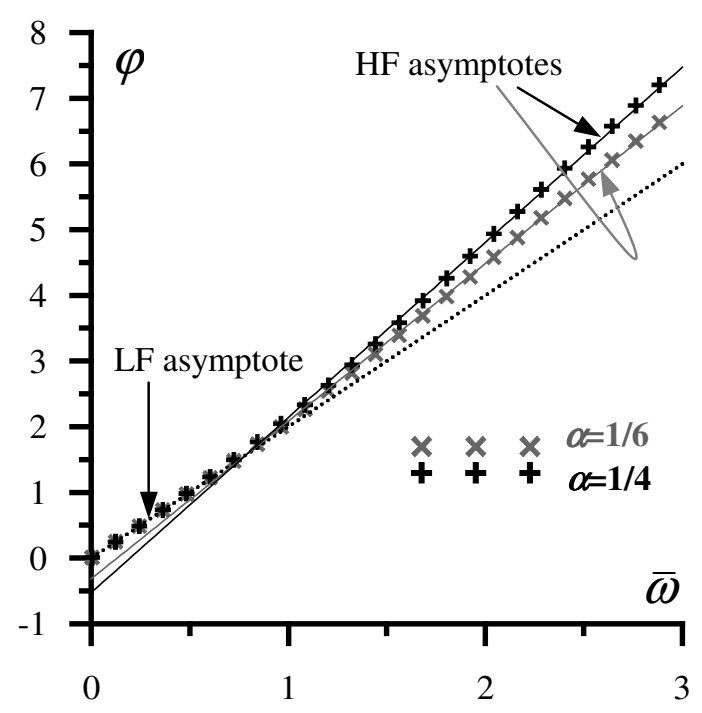

\section{Figure 2}

Dependence of the phase shift accumulated in reflection from a granular layer on dimensionless frequency $\bar{\omega}$ of the acoustic wave. The curves corresponding to the general solution (26) are indicated by " $\times$ " and " + " symbols for $\alpha=1 / 6$ and $\alpha=1 / 4$, respectively. The straight lines are low-frequency (see formula (27)) and high-frequency (see formula (28)) asymptotes. 


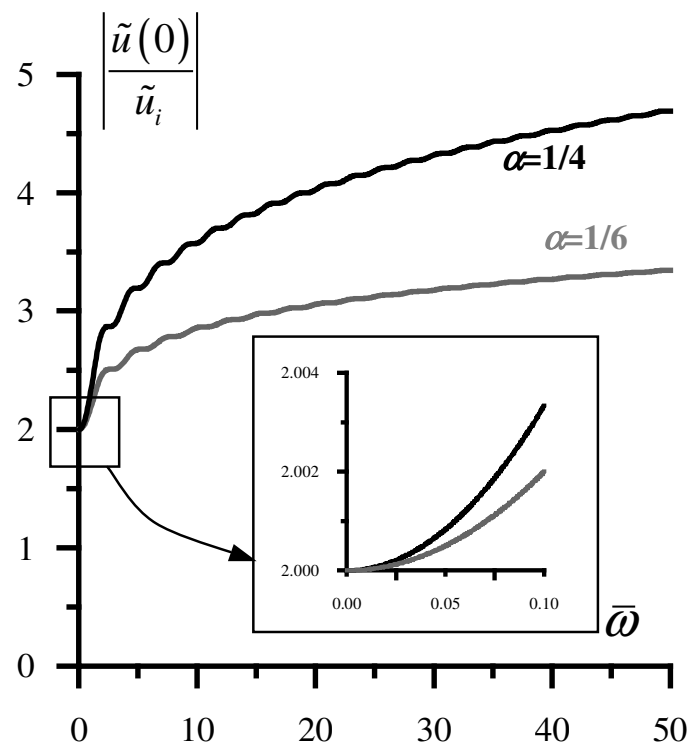

Figure 3

Dependence of the normalized surface displacement on the normalized frequency of the incident acoustic wave for two different profiles of in-depth velocity distribution $\left(c(y) \propto y^{\alpha}\right.$, $\alpha=1 / 6,1 / 4)$ 


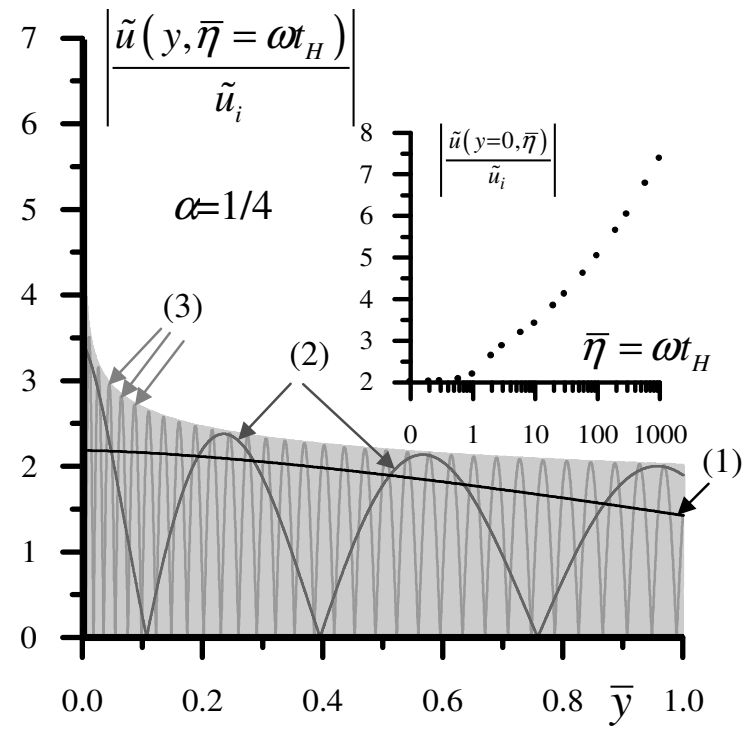

\section{Figure 4}

Distribution of the amplitude of the fundamental frequency oscillation in the depth of the granular layer (with the normalized thickness $\bar{y}=1$ ) for different normalized acoustic frequencies $\bar{\eta}=\omega t_{H}$ and $\alpha=1 / 4\left((1)-\omega t_{H}=1,(2)-\omega t_{H}=10,(3)-\omega t_{H}=10^{2}\right.$, the boundary of a region shaded in gray corresponds to amplitude of oscillation for $\omega t_{H}=10^{3}$ ). The inset illustrates the dependence of the normalized surface displacement on frequency. 


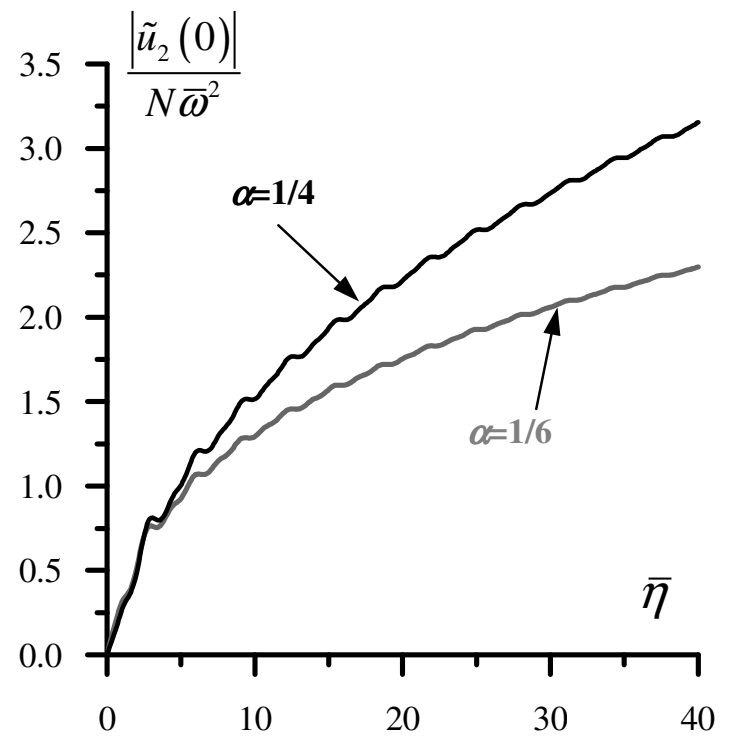

\section{Figure 5}

Dependence of the amplitude of the second harmonic $\left|\tilde{u}_{2}(0)\right|$ of the longitudinal wave at the surface on the normalized thickness $\bar{\eta}$ of the granular layer for two different powers $\alpha$ of the depth profile of sound velocity. 


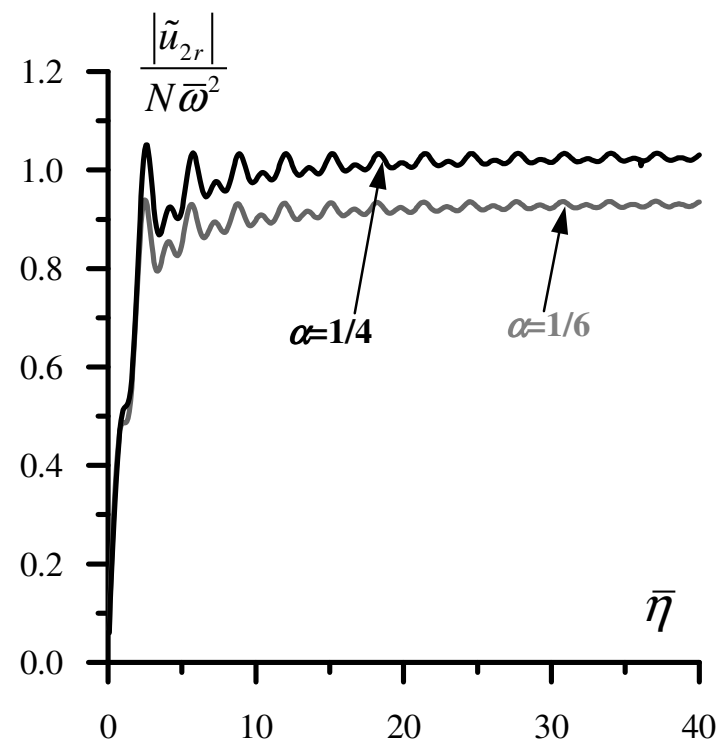

Figure 6

Dependence of the second harmonics amplitude $\left|\tilde{u}_{2 r}\right|$ of the longitudinal wave emitted into the substrate on the normalized thickness $\bar{\eta}$ of the granular layer. 


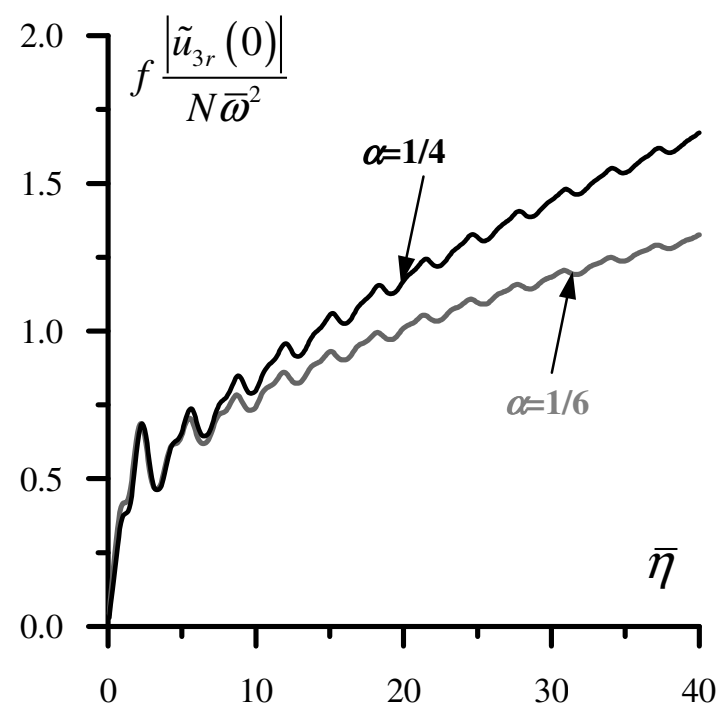

\section{Figure 7}

Dependence of the third harmonics amplitude $\left|\tilde{u}_{3}(0)\right|$ of the shear wave at the surface on the normalized thickness $\bar{\eta}$ of the granular layer for two different powers $\alpha$ of the depth profile of sound velocity. 


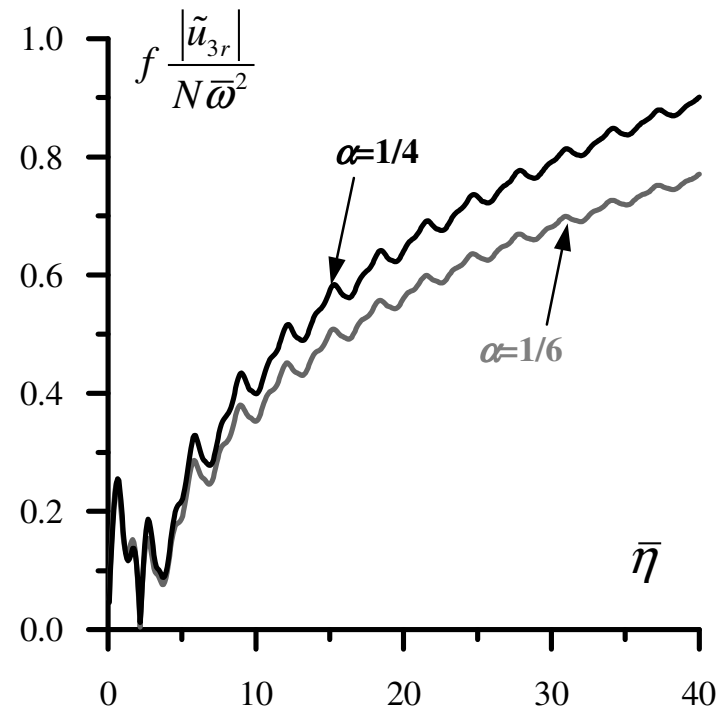

Figure 8

Dependence of the third harmonics amplitude $\left|\tilde{u}_{3 r}\right|$ of the shear wave emitted into the substrate on the normalized thickness $\bar{\eta}$ of the granular layer. 